PROCEEDINGS OF THE

AMERICAN MATHEMATICAL SOCIETY

Volume 125, Number 9, September 1997, Pages 2733-2742

S 0002-9939(97)03830-6

\title{
SIMPLY CONNECTED NILPOTENT LIE GROUPS WITH QUASI-STANDARD $C^{*}$-ALGEBRAS
}

\author{
ROBERT J. ARCHBOLD AND EBERHARD KANIUTH
}

(Communicated by Palle E. T. Jorgensen)

\begin{abstract}
The problem of when the group $C^{*}$-algebra of a locally compact group is quasi-standard is investigated for certain simply connected nilpotent Lie groups. The characterization is in terms of the coadjoint orbit structure in the dual of the Lie algebra.
\end{abstract}

\section{INTRODUCTION AND RESULTS}

A $C^{*}$-algebra $A$ is said to be quasi-standard if the failure of separation by open sets defines an open equivalence relation on the primitive ideal space $\operatorname{Prim}(A)$. This condition is a natural substitute for the stronger condition that $\operatorname{Prim}(A)$ should be Hausdorff. In the separable case, quasi-standardness of $A$ is equivalent to $A$ being representable as a maximal full algebra of cross-sections over a locally compact Hausdorff space, the complete regularization $\operatorname{Glimm}(A)$ of $\operatorname{Prim}(A)$, such that the fibres are primitive throughout a dense subset [3]. The question of which $C^{*}$ algebras can be represented in such a way has long been a matter of interest $[9,12$, 19].

Group $C^{*}$-algebras provide some of the most interesting and important examples in the theory of operator algebras. In particular, the discrete and the continuous Heisenberg groups are both known to have quasi-standard $C^{*}$-algebras (see $[1$, $20,2,3]$ ). However, the first systematic study of quasi-standardness for the $C^{*}$ algebra of a locally compact group was only recently undertaken in [18], where it was shown that $C^{*}(G)$ is quasi-standard for every amenable discrete group, while this fails to be true for the classical motion groups (except in dimension two) and other connected Lie groups. In this note, we continue the study of quasi-standard group $C^{*}$-algebras by considering the situation for some classes of simply connected nilpotent Lie groups.

For an arbitrary $C^{*}$-algebra $A$, the property of quasi-standardness can be characterized in terms of the topologized ideal spaces $\operatorname{Glimm}(A)$ and $\operatorname{Min}-\operatorname{Primal}(A)$. Here $\operatorname{Glimm}(A)$ carries the quotient topology $\tau_{q}$ of $\operatorname{Prim}(A)$ defined by the complete regularization map, whereas $\operatorname{Min}-\operatorname{Primal}(A)$, the set of minimal primal ideals of $A$, is endowed with the strong topology $\tau$. To be precise, $A$ is quasi-standard if and only if $\operatorname{Glimm}(A)$ and $\operatorname{Min}-\operatorname{Primal}(A)$ coincide as topological spaces [3]. For $\operatorname{Glimm}(A)$ and $\operatorname{Min}-\operatorname{Primal}(A)$ to agree as sets it is sufficient that every Glimm

Received by the editors November 21, 1995 and, in revised form, March 12, 1996.

1991 Mathematics Subject Classification. Primary 22D25; Secondary 22E25, 22D10.

The authors were supported by a British-German ARC Grant. 
ideal is primal. For general $C^{*}$-algebras it can easily happen that every Glimm ideal is primal but $\tau_{q} \neq \tau$. Thus, it is somewhat surprising to discover that in the case of a simply connected 2-step nilpotent Lie group $G$, if every Glimm ideal is primal, then automatically $C^{*}(G)$ is quasi-standard, whilst this does not remain true for 3-step nilpotent groups.

Our main result is the following

Theorem. Let $G$ be a simply connected nilpotent Lie group, $\mathfrak{g}$ the Lie algebra of $G$ and $\mathfrak{z}$ the centre of $\mathfrak{g}$.

(i) Suppose that the maximal coadjoint orbit dimension in $\mathfrak{g}^{*}$ equals $\operatorname{dim}(\mathfrak{g} / \mathfrak{z})$. Then $C^{*}(G)$ is quasi-standard.

(ii) Suppose that $G$ is 2-step nilpotent. Then the following conditions are equivalent.

(a) $C^{*}(G)$ is quasi-standard.

(b) The Glimm ideal contained in $\operatorname{ker} 1_{G}$ is primal.

(c) The maximal coadjoint orbit dimension in $\mathfrak{g}^{*}$ equals $\operatorname{dim}(\mathfrak{g} / \mathfrak{z})$.

For $n \geq 3$, let $\mathfrak{g}_{n}$ denote the $n$-dimensional nilpotent Lie algebra with basis $X_{1}, \ldots, X_{n}$ and non-trivial Lie brackets

$$
\left[X_{n}, X_{n-1}\right]=X_{n-2},\left[X_{n}, X_{n-2}\right]=X_{n-3}, \ldots,\left[X_{n}, X_{2}\right]=X_{1},
$$

and let $G_{n}=\exp _{\mathfrak{g}_{n}}$ be the associated simply connected Lie group. Then $G_{n}$ is $(n-1)$-step nilpotent and a semi-direct product of $\mathbb{R}$ with $\mathbb{R}^{n-1}$. The group $G_{3}$ is the continuous Heisenberg group and $G_{4}$ is the only 4-dimensional simply connected nilpotent Lie group which does not decompose into the direct product of two smaller dimensional Lie groups.

Proposition. (i) Every Glimm ideal in $C^{*}\left(G_{4}\right)$ is primal, but $C^{*}\left(G_{4}\right)$ fails to be quasi-standard.

(ii) $C^{*}\left(G_{5}\right)$ has a non-primal Glimm ideal.

It seems likely that for all $n \geq 5, C^{*}\left(G_{n}\right)$ has a Glimm ideal which is not primal. However, we have been unable to verify this.

The proofs of the theorem and the proposition are given in Sections 2 and 3, respectively. Lemmas 1 and 2 of Section 2 are likely to be useful for other classes of groups as well. In Section 4 we present further examples which, together with the theorem and the proposition, support the conjecture that for a simply connected nilpotent Lie group $G, C^{*}(G)$ is quasi-standard (if and) only if the maximal coadjoint orbit dimension in $\mathfrak{g}^{*}$ equals $\operatorname{dim}(\mathfrak{g} / \mathfrak{z})$.

\section{Proof of the Theorem}

We begin by recalling some necessary notation and basic facts concerning certain ideal spaces of $C^{*}$-algebras. For all this, compare [2] and [3]. Let $A$ be a $C^{*}$-algebra, and let $\operatorname{Id}(A)$ denote the set of all closed ideals of $A$. An ideal $I \in \operatorname{Id}(A)$ is called primal if whenever $J_{1}, \ldots, J_{n} \in \operatorname{Id}(A), n \in \mathbb{N}$, are such that $J_{1} \cdots J_{n}=\{0\}$, then $J_{k} \subseteq I$ for at least one $k$. Let $\operatorname{Min}-\operatorname{Primal}(A)$ be the set of all minimal primal ideals. Minimal primal ideals exist and can be found within any primal ideal. Min-Primal $(A)$ is endowed with the strong topology $\tau$, which makes all the functions $I \rightarrow\|a+I\|, a \in A$, continuous.

The Glimm ideal space of $A, \operatorname{Glimm}(A)$, arises from the complete regularization [9] of the primitive ideal space $\operatorname{Prim}(A)$. Define an equivalence relation $\approx$ on 
$\operatorname{Prim}(A)$ by $P \approx Q$ if $f(P)=f(Q)$ for all bounded continuous functions $f$ on $\operatorname{Prim}(A)$. Denoting by $[P]$ the equivalence class of $P$, there is a bijection between the quotient space $\operatorname{Prim}(A) / \approx$ and the so-called Glimm ideals, given by the assignment $[P] \rightarrow k([P])=\bigcap\{Q \in[P]\}$. Glimm $(A)$ carries the quotient topology $\tau_{q}$ obtained from $\operatorname{Prim}(A)$.

For $P, Q \in \operatorname{Prim}(A)$, let $P \sim Q$ if $P$ and $Q$ cannot be separated by open sets in $\operatorname{Prim}(A) . \quad A$ is called quasi-standard if $\sim$ defines an open equivalence relation on $\operatorname{Prim}(A)$. By [3, Theorem 3.3], $A$ is quasi-standard if and only if $\operatorname{Glimm}(A)$ and $\operatorname{Min}-\operatorname{Primal}(A)$ coincide as sets and topologically.

Next, we turn to representation theory. As is customary, we shall use the same letter, for example $\pi$, to denote a unitary representation of a locally compact group $G$ and the associated *-representation of the group $C^{*}$-algebra $C^{*}(G)$. Then $\operatorname{ker} \pi$ will denote the $C^{*}$-kernel of $\pi$ and $\pi \rightarrow \operatorname{ker} \pi$ defines a mapping from the dual space $\widehat{G}$ of $G$ onto $\operatorname{Prim}\left(C^{*}(G)\right)$. If $\pi$ and $\rho$ are unitary representations of $G$, then $\pi$ is weakly contained in $\rho(\pi \prec \rho)$ if $\operatorname{ker} \pi \supseteq \operatorname{ker} \rho$, and $\pi$ and $\rho$ are weakly equivalent $(\pi \sim \rho)$ if $\pi \prec \rho$ and $\rho \prec \pi$ (see [11] and [13]). For a closed subgroup $H$ of $G$ and a representation $\sigma$ of $H, \operatorname{ind}_{H}^{G} \sigma$ denotes the representation of $G$ induced by $\sigma$. By [15, Theorem 5.1], amenability of $G$ is equivalent to the property that $\pi \prec \operatorname{ind}_{H}^{G}(\pi \mid H)$ for all representations $\pi$ of $G$ and closed subgroups $H$ of $G$. Also, recall that nilpotent groups are amenable (see [14]).

Lemma 1. Let $G$ be an amenable locally compact group with centre $Z$, and for $\lambda \in \widehat{Z}$ let $I_{\lambda}=\operatorname{ker}\left(\operatorname{ind}_{Z}^{G} \lambda\right)$. Suppose that there exists a dense subset $D$ of $\widehat{Z}$ such that $I_{\lambda} \in \operatorname{Prim}\left(C^{*}(G)\right)$ for each $\lambda \in D$. Then $C^{*}(G)$ is quasi-standard and the mapping $\lambda \rightarrow I_{\lambda}$ is a homeomorphism between $\widehat{Z}$ and $\operatorname{Glimm}\left(C^{*}(G)\right)$.

Proof. For every $\pi \in \widehat{G}$, there exists a unique character $\lambda_{\pi} \in \widehat{Z}$ such that $\pi(z)=$ $\lambda_{\pi}(z) I_{\mathcal{H}_{\pi}}$ for all $z \in Z$. This defines a mapping

$$
r: \operatorname{Prim}\left(C^{*}(G)\right) \rightarrow \widehat{Z}, \quad \operatorname{ker} \pi \rightarrow \lambda_{\pi},
$$

which is well known to be surjective and continuous.

Since $G$ is amenable we have $1_{G} \prec \operatorname{ind}_{Z}^{G} 1_{Z}$ and hence

$$
\pi=\pi \otimes 1_{G} \prec \pi \otimes \operatorname{ind}_{Z}^{G} 1_{Z}=\operatorname{ind}_{Z}^{G}\left(\pi \mid Z \otimes 1_{Z}\right)=\operatorname{ind}_{Z}^{G}(\pi \mid Z) \sim \operatorname{ind}_{Z}^{G} \lambda_{\pi}
$$

for every $\pi \in \widehat{G}$. Thus

$$
\operatorname{Prim}\left(C^{*}(G)\right)=\bigcup_{\lambda \in \widehat{Z}} h\left(I_{\lambda}\right) \text { and } r^{-1}(\lambda)=h\left(I_{\lambda}\right),
$$

$\lambda \in \widehat{Z}$. Now, fix $\lambda \in \widehat{Z}$. By hypothesis, there exists a net $\left(\lambda_{\alpha}\right)$ in $D$ converging to $\lambda$. By continuity of inducing [13], $I_{\lambda_{\alpha}}-I_{\lambda}\left(\tau_{w}\right)$ and hence $I_{\lambda_{\alpha}} \rightarrow P$ in $\operatorname{Prim}\left(C^{*}(G)\right)$ for each $P \in h\left(I_{\lambda}\right)=r^{-1}(\lambda)$. Hence

$$
I_{\lambda}=\bigcap\left\{P: P \in r^{-1}(\lambda)\right\}
$$

is a primal ideal and in particular no two points in $r^{-1}(\lambda)$ can be separated by a continuous function on $\operatorname{Prim}\left(C^{*}(G)\right)$. It follows that

$$
\operatorname{Glimm}\left(C^{*}(G)\right)=\operatorname{Min}-\operatorname{Primal}\left(C^{*}(G)\right)=\left\{I_{\lambda}: \lambda \in \widehat{Z}\right\},
$$

as sets. 
To prove that $C^{*}(G)$ is quasi-standard, by [3, Theorem 3.3] it remains to show that the quotient map $q: \operatorname{Prim}\left(C^{*}(G)\right) \rightarrow \operatorname{Glimm}\left(C^{*}(G)\right)$ is open. We have a commutative diagram

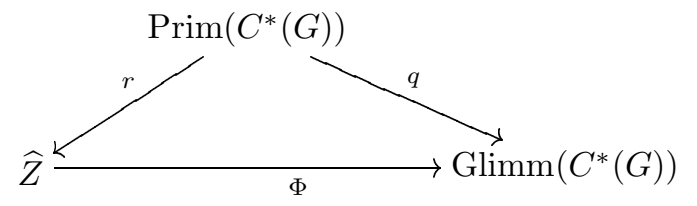

where $\Phi$ is the bijection that maps $\lambda$ to $I_{\lambda}$. Let $U$ be an open subset of $\operatorname{Prim}\left(C^{*}(G)\right)$. Since $q^{-1}(q(U))=r^{-1}(r(U))$, it is enough to show that $r^{-1}(r(U))$ is open. Towards a contradiction, suppose there is a net $\left(P_{\alpha}\right)$ in $\operatorname{Prim}\left(C^{*}(G)\right) \backslash r^{-1}(r(U))$ converging to some $P \in r^{-1}(r(U))$. Since $r$ is continuous, $r\left(P_{\alpha}\right) \rightarrow r(P)$, and since inducing is continuous,

$$
r^{-1}(r(P)) \subseteq \overline{\bigcup_{\alpha} r^{-1}\left(r\left(P_{\alpha}\right)\right)}
$$

Now, $r(P)=r(Q)$ for some $Q \in U$. Since $U$ is open, it follows from (1) that, for some $\alpha$, there exists $Q_{\alpha} \in U \cap r^{-1}\left(r\left(P_{\alpha}\right)\right)$. Then

$$
P_{\alpha} \in r^{-1}\left(r\left(Q_{\alpha}\right)\right) \subseteq r^{-1}(r(U))
$$

a contradiction.

Finally, since $r$ is continuous and open, the bijection $\Phi$ is a homeomorphism.

Lemma 2. Let $N$ be a normal subgroup of the locally compact group $G$. Suppose that $G / N$ is abelian and regard $\widehat{G / N}$ as a closed subset of $\operatorname{Prim}\left(C^{*}(G)\right)$. Let $\Gamma=$ $\left[1_{G}\right] \cap \widehat{G / N}$. Then, for any $\chi \in \widehat{G / N},[\chi] \cdot \Gamma=[\chi]$. In particular, $\Gamma$ is a closed subgroup of $\widehat{G / N}$.

Proof. Let $\chi \in \widehat{G / N}$ and let $f$ be a bounded continuous function on $\operatorname{Prim}\left(C^{*}(G)\right)$. Since $\chi$ is a character we can define a continuous function $g$ on $\operatorname{Prim}\left(C^{*}(G)\right)$ by setting

$$
g(\operatorname{ker} \pi)=f(\operatorname{ker}(\pi \otimes \chi)), \quad \pi \in \widehat{G} .
$$

Then, for every $\gamma \in \Gamma$,

$$
f(\chi \gamma)=g(\gamma)=g\left(1_{G}\right)=f(\chi)
$$

This shows that $\chi \gamma \in[\chi]$ and hence that $\Gamma$ is closed under multiplication. Also, replacing $\chi$ by $\bar{\chi}$, we obtain that $f(\bar{\chi} \gamma)=f(\bar{\chi})$. Thus, if $\chi \in \Gamma$, we have that $f\left(1_{G}\right)=f(\bar{\chi} \chi)=f(\bar{\chi})$ and hence that $\bar{\chi} \in \Gamma$.

Finally, $\Gamma$ is closed in $\widehat{G / N}$ since the Glimm class $\left[1_{G}\right]$ is closed in $\operatorname{Prim}\left(C^{*}(G)\right)$.

Let $G$ be a simply connected (and connected) nilpotent Lie group with Lie algebra $\mathfrak{g}$. Let $\mathfrak{g}^{*}$ denote the dual space of $\mathfrak{g}$ and $\mathfrak{g}^{*} / \mathrm{Ad}^{*}$ the orbit space of the coadjoint representation of $G$ on $\mathfrak{g}^{*}$. Kirillov's theory establishes a bijection between $\mathfrak{g}^{*} / \mathrm{Ad}^{*}$ and $\widehat{G}$. More precisely, associated to $f \in \mathfrak{g}^{*}$ is an irreducible representation $\pi_{f}$ of $G$, and $\pi_{f}$ and $\pi_{g}, g \in \mathfrak{g}^{*}$, are equivalent if and only if $g \in \operatorname{Ad}^{*}(G) f$. The Kirillov correspondence is a homeomorphism provided that $\mathfrak{g}^{*} / \mathrm{Ad}^{*}$ is endowed with the quotient topology [7]. 
Lemma 3. Let $G$ be a simply connected nilpotent Lie group and $d$ the maximal orbit dimension in $\mathfrak{g}^{*}$. Suppose that the set $S$ of all $g \in \mathfrak{g}^{*}$ such that $\operatorname{Ad}^{*}(G) g$ is a linear variety is dense in $\mathfrak{g}^{*}$, and suppose that $F$ is a linear subspace of $\mathfrak{g}^{*}$ such that $\bigcap_{f \in F} \operatorname{ker} \pi_{f}$ is a primal ideal in $C^{*}(G)$. Then $d \geq \operatorname{dim} F$.

Proof. Let $f_{1}, \ldots, f_{m}$ form a basis of $F$ and let $f_{0}=0$. Since the image of $S$ is dense in $\widehat{G}$, it contains a net which is convergent to $\pi_{f}$ for every $f \in F$ (compare the proof of $[2,4.6])$. Hence there is a sequence $\left(g_{n}\right)_{n}$ in $S$ such that $\pi_{g_{n}} \rightarrow \pi_{f_{j}}$ for $0 \leq j \leq m$. Since each $\operatorname{Ad}^{*}(x)$ is a homeomorphism of $\mathfrak{g}^{*}$, the quotient map $\mathfrak{g}^{*} \rightarrow \mathfrak{g}^{*} / \mathrm{Ad}^{*}$ is open. Hence there exist a subsequence $\left(g_{n_{k}}\right)_{k}$ of $\left(g_{n}\right)_{n}$ and elements $x_{j, k} \in G, 0 \leq j \leq m, k \in \mathbb{N}$, such that

$$
\operatorname{Ad}^{*}\left(x_{j, k}\right) g_{n_{k}} \rightarrow f_{j}
$$

as $k \rightarrow \infty$, for each $0 \leq j \leq m$. Thus for sufficiently large $k$, $\operatorname{dim} \operatorname{Ad}^{*}(G) g_{n_{k}} \geq m$ and hence $d \geq m=\operatorname{dim} F$.

We now recall from [4] and [6] the definition of the cortex, $\operatorname{cor}(G)$, of a locally compact group $G$. This is the closed subset of $\widehat{G}$ consisting of all those $\pi \in \widehat{G}$ that cannot be separated by open sets from the trivial representation. Clearly, $\{\operatorname{ker} \pi: \pi \in \operatorname{cor}(G)\}$ is contained in the Glimm class $\left[\right.$ ker $\left.1_{G}\right]$ of $\operatorname{Prim}\left(C^{*}(G)\right)$.

Proof of the Theorem. (i) The hypothesis implies that there exists a Zariski open and hence dense subset $F$ of $\mathfrak{g}^{*}$ such that $\operatorname{Ad}^{*}(G) f=f+\mathfrak{z}^{\perp}$ for all $f \in F$. This equality implies that $\pi_{f} \sim \operatorname{ind}_{Z}^{G}\left(\pi_{f \mid \mathfrak{z}}\right)$. Indeed,

$$
\pi_{f} \prec \operatorname{ind}_{Z}^{G}\left(\pi_{f} \mid Z\right) \sim \operatorname{ind}_{Z}^{G}\left(\pi_{f \mid \mathfrak{z}}\right)
$$

and if $g \in \mathfrak{g}^{*}$ is such that $\pi_{g} \prec \operatorname{ind}_{Z}^{G}\left(\pi_{f \mid \mathfrak{z}}\right)$, then $g|\mathfrak{z}=f| \mathfrak{z}$ and hence

$$
\operatorname{Ad}^{*}(G) g \subseteq f+\mathfrak{z}^{\perp}=\operatorname{Ad}^{*}(G) f,
$$

so that $\pi_{g}=\pi_{f}$. Of course, the set of all $f \mid \mathfrak{z}, f \in F$, is dense in $\mathfrak{z}^{*}$. Thus $G$ satisfies the conditions of Lemma 1 and this proves that $C^{*}(G)$ is quasi-standard.

(ii) In view of (i) it only remains to show that (b) implies (c). Let $Z$ denote the centre of $G$ and recall first that since $G$ is 2-step nilpotent, by [16, Lemma $6] \operatorname{cor}(G)$ coincides with the support of the conjugation representation $\gamma_{G}$ of $G$. Clearly, the support of $\gamma_{G}$ separates the points of $G / Z$. Applying Lemma 2 to $N=Z$ yields that $\widehat{G / Z}$ is contained in and hence equals the Glimm class of ker $1_{G}$ in $\operatorname{Prim}\left(C^{*}(G)\right)$. By hypothesis the Glimm ideal

$$
I_{1_{Z}}=\bigcap_{f \in \mathfrak{z}^{\perp}} \operatorname{ker} \pi_{f}
$$

is primal. Lemma 3 now gives that the maximal coadjoint orbit dimension in $\mathfrak{g}^{*}$ equals $\operatorname{dim}(\mathfrak{g} / \mathfrak{z})$.

\section{Proof of the Proposition}

Parts (i) and (ii) of the proposition are verified in the next two lemmas.

Lemma 4. Let $\mathfrak{g}_{4}$ and $G_{4}$ be as in Section 1. Then every Glimm ideal in $C^{*}\left(G_{4}\right)$ is primal, but $C^{*}\left(G_{4}\right)$ fails to be quasi-standard. 
Proof. For simplicity, write $G=G_{4}$ and $\mathfrak{g}=\mathfrak{g}_{4}$, and let $f_{1}, \ldots, f_{4}$ be the basis of $\mathfrak{g}^{*}$ which is dual to the basis $X_{1}, \ldots, X_{4}$ of $\mathfrak{g}$.

To start with, recall from [21] the parametrization of $\widehat{G}$. For $r, s, t \in \mathbb{R}, r \neq 0$, $s \neq 0$, let

$$
\sigma_{r, t}=\pi_{r f_{1}+t f_{3}} \quad \text { and } \quad \sigma_{s}=\pi_{s f_{2}} .
$$

Then, with $H=\exp \left(\mathbb{R} X_{1}+\mathbb{R} X_{2}\right)$, the commutator subgroup of $G$,

$$
\widehat{G}=\widehat{G / H} \cup\left\{\sigma_{r, t}: r, t \in \mathbb{R}, r \neq 0\right\} \cup\left\{\sigma_{s}: s \in \mathbb{R}, s \neq 0\right\} .
$$

We need the following four facts concerning the topology on $\widehat{G}$.

(1) $\widehat{G / H}$ is a limit set in $\widehat{G}$.

(2) $\left\{\sigma_{s}, \sigma_{-s}\right\}$ is a limit set in $\widehat{G}$.

(3) Each $\sigma_{r, t}$ can be separated from any different point in $\widehat{G}$ by a continuous function on $\widehat{G}$.

(4) $\sigma_{s}$ can be separated from every other point except $\sigma_{-s}$ by a continuous function on $\widehat{G}$.

To see (1), let $g_{n}=\frac{1}{n} f_{2}, n \in \mathbb{N}$. Then, for any $a, b \in \mathbb{R}$,

$$
\operatorname{Ad}^{*}((0,0,-n a, n b)) g_{n}=\frac{1}{n} f_{2}+a f_{3}+b f_{4} \rightarrow a f_{3}+b f_{4}
$$

as $n \rightarrow \infty$. Thus $\sigma_{\frac{1}{n}} \rightarrow \alpha$ for every $\alpha \in \widehat{G / H}$. For (2), choose

$n \in \mathbb{N}$. Then

$$
g_{n}=\frac{1}{n} f_{1}-\frac{1}{2} n s^{2} f_{3} \in \mathfrak{g}^{*},
$$

$$
\operatorname{Ad}^{*}((0,0,0,-n s)) g_{n}=\frac{1}{n} f_{1}+s f_{2} \text { and } \operatorname{Ad}^{*}((0,0,0, n s)) g_{n}=\frac{1}{n} f_{1}-s f_{2},
$$

so that $\sigma_{\frac{1}{n},-\frac{1}{2} n s^{2}}$ converges to both $\sigma_{s}$ and $\sigma_{-s}$.

Now, let $\mathfrak{Z}(\mathfrak{g})$ denote the centre of the universal enveloping algebra $\mathfrak{U}(\mathfrak{g})$ of $\mathfrak{g}$. For $\tau \in \widehat{G}$ let $\chi_{\tau}$ be the infinitesimal character of $\tau$. Notice that for every $a \in \mathfrak{Z}(\mathfrak{g})$, $\tau \rightarrow \chi_{\tau}(a)$ is a continuous function on $\widehat{G}$ ([5], for a simple argument compare the proof of Proposition 2.2 in [4]). By [10, Proposition 2]

$$
\mathfrak{Z}(\mathfrak{g})=\mathbb{R}\left[t_{1}, 2 t_{1} t_{3}-t_{2}^{2}\right],
$$

and by $[10$, Proposition 4]

$$
\begin{array}{ccc}
\chi_{\sigma_{r, t}}\left(t_{1}\right) & =2 \pi i r, \quad & \chi_{\sigma_{r, t}}\left(2 t_{1} t_{3}-t_{2}^{2}\right)=-8 \pi r t, \\
\chi_{\sigma_{s}}\left(t_{1}\right)=0 & \text { and } \quad & \chi_{\sigma_{s}}\left(2 t_{1} t_{3}-t_{2}^{2}\right)=4 \pi^{2} s^{2} .
\end{array}
$$

These formulas together with $\chi_{\tau}=0$ for all $\tau \in \widehat{G / H}$ and the continuity of $\tau \rightarrow$ $\chi_{\tau}(a)$ on $\widehat{G}$ for $a \in \mathfrak{Z}(\mathfrak{g})$ imply (3) and (4). Finally, combining (1), (2), (3) and (4), we obtain that, as sets,

$$
\begin{aligned}
\operatorname{Min}-\operatorname{Primal}\left(C^{*}(G)\right)= & \operatorname{Glimm}\left(C^{*}(G)\right) \\
= & \left\{\operatorname{ker} \sigma_{r, t}: r, t \in \mathbb{R}, r \neq 0\right\} \\
& \cup\left\{\operatorname{ker} \sigma_{s} \cap \operatorname{ker} \sigma_{-s}: s \in \mathbb{R}, s \neq 0\right\} \cup\left\{\operatorname{kerind}_{H}^{G} 1_{H}\right\} .
\end{aligned}
$$

To prove that $C^{*}(G)$ is not quasi-standard, by [3, Theorem 3.3] it suffices to show that the quotient map $q: \widehat{G} \rightarrow \operatorname{Glimm}\left(C^{*}(G)\right)$ fails to be open. To that end, let $C=C_{1} \cup C_{2}$ with

$$
C_{1}=\left\{\sigma_{r, 0} ; r>0\right\} \quad \text { and } \quad C_{2}=\left\{\alpha_{a, b} ; a \geq 0\right\},
$$


where for $a, b \in \mathbb{R}, \alpha_{a, b}$ is the character of $G / H=\mathbb{R}^{2}$ corresponding to $a, b$. We claim that $C$ is closed in $\widehat{G}$.

Notice first that $(r, t) \rightarrow \sigma_{r, t}$ is a homeomorphism between $(\mathbb{R} \backslash\{0\}) \times \mathbb{R}$ and $\widehat{G} \backslash(G / Z(G))$, so that $C_{1}$ is closed in $\widehat{G} \backslash(G / Z(G))$. If $\sigma_{r, 0} \rightarrow \sigma_{s}$ for some $s$, then

$$
r \rightarrow 0, \quad-x_{4} r \rightarrow s \quad \text { and } \quad \frac{1}{2} x_{4}^{2} r \rightarrow 0
$$

(compare the description of the coadjoint orbits in [21]), which is impossible. If $\sigma_{r, 0} \rightarrow \alpha_{a, b}$, then $\frac{1}{2 r}\left(x_{4} r\right)^{2} \rightarrow a$, so that $a \geq 0$.

Thus $U=\widehat{G} \backslash C$ is open in $\widehat{G}$. However,

$$
\widehat{G} \backslash q^{-1}(q(U))=\left\{\sigma_{r, 0}: r>0\right\}
$$

is not closed in $\widehat{G}$ since its closure contains $1_{G}$.

Lemma 5. $C^{*}\left(G_{5}\right)$ has a non-primal Glimm ideal.

Proof. Let $G=G_{5}$ and $\mathfrak{g}=\mathfrak{g}_{5}$, and let $f_{1}, \ldots, f_{5}$ be the basis of $\mathfrak{g}^{*}$ which is dual to the basis $X_{1}, \ldots, X_{5}$. Towards a contradiction, let us suppose that the unique Glimm ideal contained in $\operatorname{ker} 1_{G}$ is primal. Then there is a net in $\widehat{G}$ converging to every element in $\operatorname{cor}(G)$. By $[6]$,

$$
\operatorname{cor}(G)=\left\{\pi_{g}: g=\sum_{j=3}^{5} \eta_{j} f_{j}, \eta_{j} \in \mathbb{R}\right\} .
$$

Let $c$ and $d$ be distinct non-zero real numbers. Then there is a sequence $\left(g_{n}\right)_{n}$ in $\mathfrak{g}^{*}$ such that

$$
\pi_{g_{n}} \rightarrow 1_{g}, \quad \pi_{g_{n}} \rightarrow \pi_{c f_{3}} \quad \text { and } \quad \pi_{g_{n}} \rightarrow \pi_{d f_{3}} .
$$

Choosing different orbit representatives and by passing to a subsequence if necessary, we may assume that $g_{n} \rightarrow 0$ and may find sequences $\left(x_{n}\right)$ and $\left(y_{n}\right)$ in $G$ such that

$$
\operatorname{Ad}^{*}\left(x_{n}\right) g_{n} \rightarrow c f_{3} \quad \text { and } \quad \operatorname{Ad}^{*}\left(y_{n}\right) g_{n} \rightarrow d f_{3}
$$

as $n \rightarrow \infty$. The coadjoint orbit of $g=\sum_{j=1}^{5} \xi_{j} f_{j}$ consists of points

$$
\xi_{1} f_{1}+\left(\xi_{2}+t \xi_{1}\right) f_{2}+\left(\xi_{3}+t \xi_{2}+\frac{1}{2} t^{2} \xi_{1}\right) f_{3}+\left(\xi_{4}+t \xi_{3}+\frac{1}{2} t^{2} \xi_{2}+\frac{1}{6} t^{3} \xi_{1}\right) f_{4}+s f_{5},
$$

$t, s \in \mathbb{R}[21]$. Thus if we write $g_{n}=\sum_{j=1}^{5} \xi_{j, n} f_{j}, n \in \mathbb{N}$, there are sequences $\left(t_{n}\right)$ and $\left(s_{n}\right)$ in $\mathbb{R}$ such that

$$
\begin{gathered}
\xi_{3, n}+t_{n} \xi_{2, n}+\frac{1}{2} t_{n}^{2} \xi_{1, n} \rightarrow c, \\
\xi_{4, n}+t_{n} \xi_{3, n}+\frac{1}{2} t_{n}^{2} \xi_{2, n}+\frac{1}{6} t_{n}^{3} \xi_{1, n} \rightarrow 0, \\
\xi_{3, n}+s_{n} \xi_{2, n}+\frac{1}{2} s_{n}^{2} \xi_{1, n} \rightarrow d, \\
\xi_{4, n}+s_{n} \xi_{3, n}+\frac{1}{2} s_{n}^{2} \xi_{2, n}+\frac{1}{6} s_{n}^{3} \xi_{1, n} \rightarrow 0 .
\end{gathered}
$$

Since $\xi_{j, n} \rightarrow 0$, (1) yields $\left|t_{n}\right| \rightarrow \infty$. From (1) we get

$$
\frac{1}{2} t_{n}^{2} \xi_{1, n}=c-t_{n} \xi_{2, n}+\varepsilon_{n}
$$

where $\varepsilon_{n} \rightarrow 0$. Inserting this into (2), gives

$$
t_{n}\left(\xi_{3, n}+\frac{c}{3}+\frac{\varepsilon_{n}}{3}+\frac{1}{6} t_{n} \xi_{2, n}\right) \rightarrow 0,
$$


whence $t_{n} \xi_{2, n} \rightarrow-2 c$. Hence $t_{n}^{2} \xi_{1, n} \rightarrow 6 c$ and therefore

$$
\frac{\xi_{2, n}^{2}}{\xi_{1, n}}=\frac{\left(t_{n} \xi_{2, n}\right)^{2}}{t_{n}^{2} \xi_{1, n}} \rightarrow \frac{2 c}{3} .
$$

Using $\left(1^{\prime}\right)$ and $\left(2^{\prime}\right)$ we obtain that

$$
\frac{\xi_{2, n}^{2}}{\xi_{1, n}}=\frac{\left(s_{n} \xi_{2, n}\right)^{2}}{s_{n}^{2} \xi_{1, n}} \rightarrow \frac{2 d}{3},
$$

contradicting the fact that $c \neq d$.

\section{FURTHER EXAMPLES}

We conclude this note by presenting several examples which, on the one side, indicate that it will be difficult to find, for arbitrary simply connected nilpotent Lie groups $G$, simple conditions on $G$ itself for quasi-standardness of $C^{*}(G)$ and for $\operatorname{Glimm}\left(C^{*}(G)\right)$ and Min-Primal $\left(C^{*}(G)\right)$ to coincide just as sets. However, together with the theorem and the proposition, these examples lead to the conjecture that $C^{*}(G)$ is quasi-standard only if the maximal coadjoint orbit dimension $\mathfrak{g}^{*}$ equals the dimension of $\mathfrak{g} / \mathfrak{z}$.

First we discuss the five-dimensional simply connected nilpotent Lie groups.

Example 1. Up to topological isomorphism there are exactly six simply connected nilpotent Lie groups of dimension 5 which cannot be written as direct products of smaller-dimensional ones. Using the data provided in [21] we now check which of these groups have quasi-standard $C^{*}$-algebras. For any simply connected nilpotent Lie group $G$ with Lie algebra $\mathfrak{g}$ let $d(G)$ denote the maximal coadjoint orbit dimension in $\mathfrak{g}^{*}$.

Retaining the notations of [21], observe first that

$$
d\left(G_{5,1}\right)=d\left(G_{5,3}\right)=d\left(G_{5,6}\right)=4 .
$$

Thus $C^{*}\left(G_{5,1}\right), C^{*}\left(G_{5,3}\right)$ and $C^{*}\left(G_{5,6}\right)$ are quasi-standard by (i) of the theorem. Next, $G_{5,2}$ is 2-step nilpotent with 2-dimensional centre and $d\left(G_{5,2}\right)=2$, so that by the theorem there is a Glimm ideal in $C^{*}\left(G_{5,2}\right)$ which is not primal. With the notation from the previous section, $G_{5,5}=G_{5}$. Hence by Lemma 5 , there exists a Glimm ideal in $C^{*}\left(G_{5,5}\right)$ which is not primal.

The remaining case, $G_{5,4}$, is much more complicated though similar to $G_{4}$. We therefore only give a sketch of the proof that $C^{*}\left(G_{5,4}\right)$ fails to be quasi-standard. Let $G=G_{5,4}$ and $\mathfrak{g}=\mathfrak{g}_{5,4}$, and for $r, s, t, u, v \in \mathbb{R}$ set

$$
\sigma_{r, s, t, u, v}=\pi_{r f_{1}+s f_{2}+t f_{3}+u f_{4}+v f_{5}} \in \widehat{G},
$$

where $f_{1}, \ldots, f_{5}$ is the basis of $\mathfrak{g}^{*}$ dual to the basis $X_{1}, \ldots, X_{5}$ of $\mathfrak{g}$. The infinitesimal characters for irreducible representations of $G$ have been determined in [10, Proposition 8]. It follows that each point in

$$
S=\left\{\sigma_{r, s, t, u, v}: r \neq 0 \text { or } s \neq 0\right\}
$$

can be separated from every distinct point in $\widehat{G}$ by a continuous function. Set

$$
C_{1}=\left\{\sigma_{r, 0,0,0,0}: r>0\right\} \quad \text { and } \quad C_{2}=\left\{\sigma_{0,0,0, u, v}: v \geq 0\right\} .
$$

$C_{2}$ is closed in $\widehat{G}$ and $C_{1}$ is closed in $S$. Examining the coadjoint orbit structure in $\mathfrak{g}^{*}$ (see [21]), it can easily be verified that no $\sigma_{0,0, t, u, v}$ with $t \neq 0$ and no $\sigma_{0,0,0, u, v}$ with 
$v<0$ can be the limit of a net in $C_{1}$. Hence $\bar{C}_{1} \subseteq C_{1} \cup C_{2}$. Thus $U=\widehat{G} \backslash\left(C_{1} \cup C_{2}\right)$ is open in $\widehat{G}$. Observe also that

$$
\widehat{G / G^{\prime}}=\left\{\sigma_{0,0,0, u, v}: u, v \in \mathbb{R}\right\}
$$

is a limit set in $\widehat{G}$. With $q: \widehat{G} \rightarrow \operatorname{Glimm}\left(C^{*}(G)\right)$ denoting the quotient map, it follows as for $G_{4}$ that $q(U)$ is not open in $\operatorname{Glimm}\left(C^{*}(G)\right)$. Therefore $C^{*}(G)$ is not quasi-standard.

Every non-abelian 5-dimensional simply connected nilpotent Lie group $G$ which is a direct product of smaller-dimensional ones is isomorphic to either $G_{3} \times \mathbb{R}^{2}$ or $G_{4} \times \mathbb{R}$. By using the properties of $C^{*}\left(G_{3}\right)$ and $C^{*}\left(G_{4}\right)$ (see Section 1) and tensor product results from [17] it is straightforward to check that $C^{*}\left(G_{3} \times \mathbb{R}^{2}\right)$ is quasi-standard, while $C^{*}\left(G_{4} \times \mathbb{R}\right)$ is not, but Glimm ideals in $C^{*}\left(G_{4} \times \mathbb{R}\right)$ are primal.

Finally we show that quasi-standardness is in general not preserved when moving to either quotients or connected normal subgroups. In fact, for quotients and connected normal subgroups, some Glimm ideals may even fail to be primal.

Example 2. Let $\mathfrak{g}$ be the nilpotent Lie algebra of dimension 7 with basis $X_{1}, \ldots, X_{7}$ and non-trivial commutators

$$
\begin{gathered}
{\left[X_{7}, X_{5}\right]=X_{1}, \quad\left[X_{6}, X_{2}\right]=X_{1}, \quad\left[X_{6}, X_{5}\right]=X_{3},} \\
{\left[X_{5}, X_{4}\right]=-X_{2} \quad \text { and } \quad\left[X_{4}, X_{3}\right]=X_{1},}
\end{gathered}
$$

and let $G=\exp \mathfrak{g}$. It is straightforward to compute the coadjoint orbits in $\mathfrak{g}^{*}$. For $x=\left(x_{1}, \ldots, x_{7}\right) \in G$ and $f=\sum_{i=1}^{7} \xi_{i} f_{i} \in \mathfrak{g}^{*}$, we have

$$
\begin{aligned}
\operatorname{Ad}^{*}(x) f= & f+x_{6} \xi_{1} f_{2}+x_{4} \xi_{1} f_{3}+\left(x_{3} \xi_{1}+x_{5} \xi_{2}\right) f_{4} \\
& +\left(x_{7} \xi_{1}+x_{4} \xi_{2}+x_{6} \xi_{3}+x_{6} x_{4} \xi_{1}\right) f_{5} \\
& +\left(x_{5} x_{4} \xi_{1}+x_{5} \xi_{3}+x_{2} \xi_{1}\right) f_{6}+x_{5} \xi_{1} f_{7} .
\end{aligned}
$$

It is obvious that $\mathfrak{z}=\mathbb{R} X_{1}$ and $\operatorname{Ad}^{*}(G) f=f+\mathfrak{z}^{\perp}$ if $\xi_{1} \neq 0$. Thus, by (i) of the theorem, $C^{*}(G)$ is quasi-standard.

Now, the subalgebra of $\mathfrak{g}$ generated by $X_{1}, X_{2}, X_{3}, X_{5}, X_{7}$ is isomorphic to $\mathfrak{g}_{5,2}$, and we already know that $C^{*}\left(G_{5,2}\right)$ contains a Glimm ideal which is not primal (Example 1). Also, it is easily checked that $\mathfrak{g} / \mathfrak{z}=\mathbb{R} \oplus \mathfrak{h}$, where $\mathfrak{h}$ is isomorphic to $\mathfrak{g}_{5,2}$. Hence $C^{*}(G / Z(G))$ has a non-primal Glimm ideal.

\section{ACKNOWLEDGEMENT}

The authors are grateful to the referee for pointing out an error in an earlier version of the paper and for several helpful suggestions.

\section{ADDED IN PROOF}

In subsequent work with G. Schlichting and D. W. B. Somerset the authors have shown that $C^{*}\left(G_{n}\right)$ has a non-primal Glimm ideal for all $n \geq 5$ and that there exist quasi-standard 6-dimensional simply connected nilpotent Lie groups $G$ such that the maximal coadjoint orbit dimension is less than $\operatorname{dim}(\mathfrak{g} / \mathfrak{z})$. 


\section{REFERENCES}

1. J. Anderson and W. Paschke, The rotation algebra, Houston Math. J. 15 (1989), 1-26. MR 90k: 46126

2. R. J. Archbold, Topologies for primal ideals, J. London Math. Soc. (2) 36 (1987), 524-542. MR 89h: 46076

3. R. J. Archbold and D. W. B. Somerset, Quasi-standard $C^{*}$-algebras, Math. Proc. Camb. Phil. Soc. 107 (1990), 349-360. MR 91c:46079

4. M. B. Bekka and E. Kaniuth, Irreducible representations of locally compact groups that cannot be Hausdorff separated from the identity representation, J. Reine Angew. Math. 385 (1988), 203-220. MR 89j:22005

5. P. Bernat and J. Dixmier, Sur le dual d'un groupe de Lie, C. R. Acad. Sci. Paris 250 (1960), 1778-1779. MR 27:1536

6. J. Boidol, J. Ludwig and D. Müller, On infinitely small orbits, Studia Math. 88 (1988), 1-11. MR 89f: 22009

7. I. Brown, Dual topology of a nilpotent Lie group, Ann. Sci. Ec. Norm. Sup. 6 (1973), 407-411. MR 50:4813

8. L. Corwin and F. P. Greenleaf, Representations of nilpotent Lie groups and their applications. Part 1: Basic theory and examples, Cambridge University Press, Cambridge, 1990. MR 92b: 22007

9. J. Dauns and K. H. Hofmann, Representations of rings by continuous sections, Memoirs Amer. Math. Soc. 83, AMS 1968. MR 40:752

10. J. Dixmier, Sur les représentations unitaires des groupes de Lie nilpotents. III, Canad. J. Math. 10 (1958), 321-348. MR 20:1929

11. L Les $C^{*}$-algèbres et leurs représentations, Gauthier-Villars, Paris, 1964. MR 30:1404

12. J. M. G. Fell, The structure of algebras of operator fields, Acta Math. 106 (1961), 233-280. MR 29:1547

13. __ Weak containment and induced representations of groups. II, Trans. Amer. Math. Soc. 110 (1964), 424-447. MR 28:3114

14. F. P. Greenleaf, Invariant means on topological groups and their applications, Van Nostrand, New York, 1969. MR 40:4776

15. _ Amenable actions of locally compact groups, J. Funct. Anal. 4 (1969), 295-315. MR 40:268

16. E. Kaniuth, On the conjugation representation of a locally compact group, Math. Z. 202 (1989), 275-288. MR 90k:22003

17. - Minimal primal ideal spaces and norms of inner derivations of tensor products of $C^{*}$-algebras, Math. Proc. Camb. Phil. Soc. 119 (1996), 297-308. CMP 96:03

18. E. Kaniuth, G. Schlichting and K. F. Taylor, Minimal primal and Glimm ideal spaces of group $C^{*}$-algebras, J. Funct. Anal. 130 (1995), 43-76. CMP 95:12

19. R. Lee, On the $C^{*}$-algebras of operator fields, Indiana Univ. Math. J. 25 (1976), 303-314. MR 53:14150

20. _ Full algebras of operator fields trivial except at one point, Indiana Univ. Math. J. 26 (1977), 351-372. MR 55:3812

21. O. A. Nielsen, Unitary representations and coadjoint orbits of low-dimensional nilpotent Lie groups, Queen's Papers in Pure Appl. Math. 63 (1983). MR 86k:22020

Department of Mathematical Sciences, University of Aberdeen, Aberdeen AB24 3QY, Scotland, United Kingdom

E-mail address: r.archbold@maths.abdn.ac.uk

Fachbereich Mathematik/Informatik, Universität Paderborn, D-33095 Paderborn, GERMANY

E-mail address: kaniuth@uni-paderborn.de 